\title{
Detection of the $\mathrm{H} 92 \alpha$ recombination line from the starbursts in the Circinus galaxy and NGC 1808
}

\author{
A. L. Roy ${ }^{1,2,3,4}$, W. M. Goss ${ }^{3}$, and K. R. Anantharamaiah ${ }^{4, \star}$ \\ 1 Max-Planck-Institut für Radioastronomie, Auf dem Hügel 69, 53121 Bonn, Germany \\ e-mail: aroy@mpifr-bonn.mpg.de \\ 2 Australia Telescope National Facility, PO Box 76, Epping 1710, NSW, Australia \\ 3 NRAO, PO Box O, Socorro, NM 87801, USA \\ ${ }^{4}$ Raman Research Institute, CV Raman Ave, Sadashivanagar, Bangalore 560080, India \\ Received 5 March 2007 / Accepted 24 January 2008
}

ABSTRACT

\begin{abstract}
Context. Gas ionized by starburst activity radiates radio recombination lines (RRLs), from which one can derive the plasma conditions and the number of massive stars formed in the burst, free of dust obscuration effects.

Aims. We aimed to find detectable RRL emission from additional extragalactic starburst systems and to use the line properties to estimate the properties of the ionized gas.

Methods. We conducted a search for RRLs in the nearby extragalactic starburst or Seyfert galaxies NGC 1808, the Circinus galaxy, NGC 4038/9, II Zw 40, NGC 6221, NGC 7552, IRAS 18325-5926, IC 5063, and VV 114. We used the Very Large Array with resolution of $3^{\prime \prime}$ to 32" and the Australia Telescope Compact Array with resolution of 10" to search for the RRLs H91 $\alpha$ and H92 $\alpha$ with rest frequencies of $8.6 \mathrm{GHz}$ and $8.3 \mathrm{GHz}$. From the new detections we derive conditions in the starburst regions.

Results. We detected for the first time RRLs from the starburst nuclei in the Circinus galaxy and NGC 1808. The Circinus galaxy was detected in RRL emission with a line strength integrated over the source of $3.2 \mathrm{mJy}$, making it the fourth-strongest extragalactic RRL emitter known at this frequency (after NGC 4945, M 82, and NGC 253) and so is suitable for detailed study. The line and continuum emission from the Circinus galaxy can be matched by a model consisting of a collection of 50 to $10000 \mathrm{H}$ II regions with temperatures of $5000 \mathrm{~K}$, densities of $500 \mathrm{~cm}^{-3}$ to $50000 \mathrm{~cm}^{-3}$, and a total effective diameter of $3 \mathrm{pc}$ to $50 \mathrm{pc}$. The Lyman continuum production rate required to maintain the ionization is $1 \times 10^{52} \mathrm{~s}^{-1}$ to $3 \times 10^{53} \mathrm{~s}^{-1}$, which requires 300 to $9000 \mathrm{O} 5$ stars to be produced in the starburst, inferring a star formation rate of $0.2 M_{\odot} \mathrm{yr}^{-1}$ to $6 M_{\odot} \mathrm{yr}^{-1}$. NGC 1808 was detected in RRL emission at 3.9 $\sigma$ with a line strength of $0.47 \mathrm{mJy}$ at the expected velocity. No radio recombination lines were detected from the other galaxies surveyed to a $3 \sigma$ limit of $0.3 \mathrm{mJy}$ to $1.4 \mathrm{mJy}$.

Conclusions. We have detected RRLs from two galaxies, adding to the small but growing number of known extragalactic RRL emitters. The Circinus galaxy is strong and especially suited to high-quality follow-up spectroscopic study. We derived conditions and star formation rates in the starbursting regions. Uncertainties can be reduced by future multi-transition studies.
\end{abstract}

Key words. galaxies: individual: NGC 1808 - galaxies: nuclei - radio lines: galaxies

\section{Introduction}

Starburst activity has a profound impact on galaxies, through prodigious formation of stars and clusters, ionization of gas and outflows driven by overpressure in the starbursting region that redistribute the interstellar medium (ISM). Studies of the gas ionized in the burst are interesting because they provide information on the ionizing photon production rate and hence the number of high-mass stars formed and on the conditions in the surrounding ISM.

However, optical and infrared diagnostics of the ISM are hampered by dust obscuration associated with the molecular clouds from which the stars form. Radio studies are free of dust obscuration effects and the detection of radio recombination lines is particularly useful as their strengths provide diagnostics of the plasma conditions and give dynamical information with arcsec resolution.

RRLs were first detected from the starbursts in M 82 and NGC 253 by Shaver et al. (1977) and Seaquist \& Bell (1977) soon after the potential to detect and interpret extragalactic RRLs

* Deceased. was shown by Shaver (1978). Those galaxies have since been studied over a wide range of frequencies, giving constraints on the physical state and kinematics in the nuclear regions (e.g. Anantharamaiah \& Goss 1997; Rodríguez-Rico et al. 2006).

However, surveys to find RRLs in other galaxies produced no further detections for a period (Churchwell \& Shaver 1979; Bell \& Seaquist 1978; Bell et al. 1984). When survey sensitivities were improved by an order of magnitude using the Very Large Array (VLA) during a renewed effort in the early 1990s, RRLs were detected near $8.6 \mathrm{GHz}$ from several bright starburst galaxies. Those new detections are NGC 660, NGC 1365, NGC 2146, NGC 3628, NGC 3690, NGC 5253, M 83, IC 694, Arp 220, Henize 2-10 (Anantharamaiah et al. 1993; Zhao et al. 1996; Phookun et al. 1998; Mohan et al. 2001), and NGC 4945 at mm wavelengths (Viallefond, private communication).

During an extension of the RRL surveys to southerly starburst and Seyfert galaxies using the Australia Telescope Compact Array (ATCA), we made three new detections: NGC 3256, the Circinus galaxy, and NGC 4945. The detection of NGC 3256 was reported in a previous paper (Roy et al. 2005a). Here, we report the detection of the Circinus galaxy and four 
upper limits (NGC 6221, NGC 7552, IC 5063, and IRAS 183255926). The detection of NGC 4945 will be reported in a later paper. A short report of all three detections was published by Roy et al. (2005b).

During an extension of the RRL surveys using the VLA, we made a new detection of NGC 1808 (Mohan 2002) and established sensitive upper limits on four other galaxies (NGC 4038/9, NGC 7552, II Zw 40, and VV 114), all of which are reported here.

We adopt $H_{0}=75 \mathrm{~km} \mathrm{~s}^{-1} \mathrm{Mpc}^{-1}, q_{0}=0.5$, and $\Lambda=0$, and give velocities in the heliocentric frame using the optical velocity definition throughout this paper.

\section{Observations}

\subsection{ATCA observations}

We observed the Circinus galaxy, IC 5063, IRAS 183255926, NGC 7552, and NGC 6221 with the Australia Telescope Compact Array (ATCA). The observing parameters and results are summarized in Table 1.

Calibration and imaging were done using the AIPS software using standard methods. The flux-density scale assumed that PKS B1934-638 had a flux density of $2.99 \mathrm{Jy}$ at $8295 \mathrm{MHz}$ and $2.87 \mathrm{Jy}$ at $8570 \mathrm{MHz}$, based on the Baars et al. (1977) fluxdensity scale. A phase calibrator was observed every half hour to correct the instrumental phase response. A bandpass calibrator was observed every few hours for correcting the instrumental frequency response (bandpass). Phase corrections obtained from self calibration of the continuum source were applied to the spectral line data. Continuum emission was subtracted from the line data using the method UVLSF (Cornwell et al. 1992) in which the continuum is determined for each baseline by a linear fit to the visibility spectrum. The final continuum and line images were made using natural weighting of the $(u, v)$ data to achieve maximum possible signal-to-noise ratio and we averaged together the two transitions and two polarizations.

Uncertainties on the absolute flux densities have an $11 \% \mathrm{rms}$ random multiplicative component due to flux-density bootstrapping and atmospheric opacity, a $0.12 \mathrm{mJy}$ rms random additive component due to thermal noise in a $1 \mathrm{MHz}$ channel and a systematic multiplicative component of $11 \% \mathrm{rms}$ due mainly to the uncertainty in the Baars et al. flux-density scale.

\subsection{VLA observations}

NGC 1808, NGC 4038/9, II Zw 40, NGC 7552, and VV 114 were observed with the Very Large Array (VLA). The observing parameters and results are summarized in Table 2. Data reduction was similar to the ATCA reduction.

For NGC 1808, the CnB data suffered from a high residual bandpass calibration error causing low SNR spectra. Thus, we based the analysis on the DnC spectral data, but show the continuum image from the higher resolution $\mathrm{CnB}$ data.

\section{The Circinus galaxy}

This remarkable low-latitude spiral is at a distance of about $4 \mathrm{Mpc}$ (thus $1^{\prime \prime}$ is $19 \mathrm{pc}$ ) and is the closest Seyfert galaxy (type 1) with many signs of a starburst in the nucleus.

The H I distribution has been studied with the ATCA (Jones et al. 1999) who find a total size of about $80^{\prime}$ or $90 \mathrm{kpc}$ with a total H I mass of $7 \times 10^{9} M_{\odot}$. Detailed imaging of the circumstellar ring with a radius of about $220 \mathrm{pc}$ in $\mathrm{H} \alpha$ (Elmouttie et al. 1998a) shows a rotational speed of about $350 \mathrm{~km} \mathrm{~s}^{-1}$ in rough agreement with an H I feature (Jones et al. 1999). This feature may lie close to the inner Lindblad resonance.

A number of studies of the radio continuum have been carried out (Elmouttie et al. 1998b; Harnett et al. 1990; Elmouttie et al. 1995). The peculiar radio lobes orthogonal to the disk of the galaxy are polarized and the nuclear source (about $0.1 \mathrm{Jy}$ at $1.4 \mathrm{GHz})$ has a flat spectral index of $\alpha=-0.06\left(S \propto v^{\alpha}\right)$ with a size limit of $\sim 20 \mathrm{pc}$. Higher resolution radio images by Davies et al. (1998) at $8.4 \mathrm{GHz}$ and $5 \mathrm{GHz}$ with a resolution of $1^{\prime \prime}$ to $2^{\prime \prime}$ show a compact source with a size of about $0.6^{\prime \prime}$ or $12 \mathrm{pc}$. There is also a reported radio core of $19 \mathrm{mJy}$ at $2.3 \mathrm{GHz}$ observed with a beam of $0.1^{\prime \prime}$ with the Parkes-Tidbinbilla Interferometer, which can be associated with the AGN. Davies et al. suggest that the flat spectral index of the nucleus could arise from free-free absorption.

There have been several studies of the molecular content of the Circinus galaxy by Curran et al. $(1998,2001)$ and Elmouttie et al. (1998c). The former authors have carried out a systematic study of numerous molecules (e.g. four isotopes of $\mathrm{CO}$ in several transitions, $\mathrm{CS}, \mathrm{H}_{2} \mathrm{CO}, \mathrm{HCN}, \mathrm{HCO}^{+}$and other molecules). They propose that the $\mathrm{H}_{2}$ density near the nucleus is in the range $2 \times$ $10^{3} \mathrm{~cm}^{-3}$ to $10^{5} \mathrm{~cm}^{-3}$. The molecular emission is distributed in a disk of radius $300 \mathrm{pc}$ which is the likely source of the gas for the star formation. $\mathrm{H}_{2} \mathrm{O}$ masers have been extensively studied by Greenhill et al. (2003a) and Greenhill et al. (2003b). Doppler shifts up to $\sim 460 \mathrm{~km} \mathrm{~s}^{-1}$ are observed.

In the optical and near IR, high resolution investigations of the Circinus galaxy have been carried out by Wilson et al. (2000) who have used HST imaging in [O III], $\mathrm{H} \alpha, \mathrm{H}_{2}$ and continuum bands in the optical and the near IR. A one-sided ionization cone is observed in $\mathrm{H} \alpha$ and [O III] and the nuclear starburst activity is shown in $\mathrm{H} \alpha$ images at distances of tens of parsecs from the centre. The circumstellar star-forming ring of radius $\sim 200 \mathrm{pc}$ is prominent.

\subsection{Results}

The ATCA continuum and line images, integrated spectrum, and position-velocity diagram are shown in Figs. 1 to 3 . The measured line and continuum parameters are given in Table 1.

The continuum image shows a single extended continuum component. The continuum emission is predominantly nonthermal, with a spectral index of -0.65 between $1.4 \mathrm{GHz}$ and 8.3 GHz (Elmouttie et al. 1998b).

Line emission was detected in the nuclear region, coincident with the peak continuum emission with a tail of emission extending $25^{\prime \prime}(500 \mathrm{pc})$ towards the north-east. The total area of line emission is 1.9 beam areas, or $(250 \mathrm{pc})^{2}$.

The $\mathrm{H} 91 \alpha+\mathrm{H} 92 \alpha$ spectrum integrated over the lineemitting region shows a clear line detection with $26 \sigma$ significance with centroid at $480 \mathrm{~km} \mathrm{~s}^{-1}$, compared to the H I systemic velocity of $449 \mathrm{~km} \mathrm{~s}^{-1}$ (Juraszek et al. 2000). The line FWHM is $260 \mathrm{~km} \mathrm{~s}^{-1}$ after deconvolving the instrumental velocity resolution of $42 \mathrm{~km} \mathrm{~s}^{-1}$.

The position-velocity diagram (Fig. 3) shows a gradient in the east-west direction of $130 \mathrm{~km} \mathrm{~s}^{-1}$ over the central $25^{\prime \prime}$ $(475 \mathrm{pc})$ consistent with a disk rotating about a north-south axis with the east side receding. The position angle for the positionvelocity slice was chosen by inspecting the first-moment image, which showed the velocity gradient to be east-west. Interestingly, the gradient direction is opposite that of the $\mathrm{Br} \gamma$ or $\mathrm{H}_{2}$ (Müller Sanchez et al. 2006), the $\mathrm{H}_{2} \mathrm{O}$ masers (Greenhill et al. 2003b) and the HI disk (Jones et al. 1999), all of which 


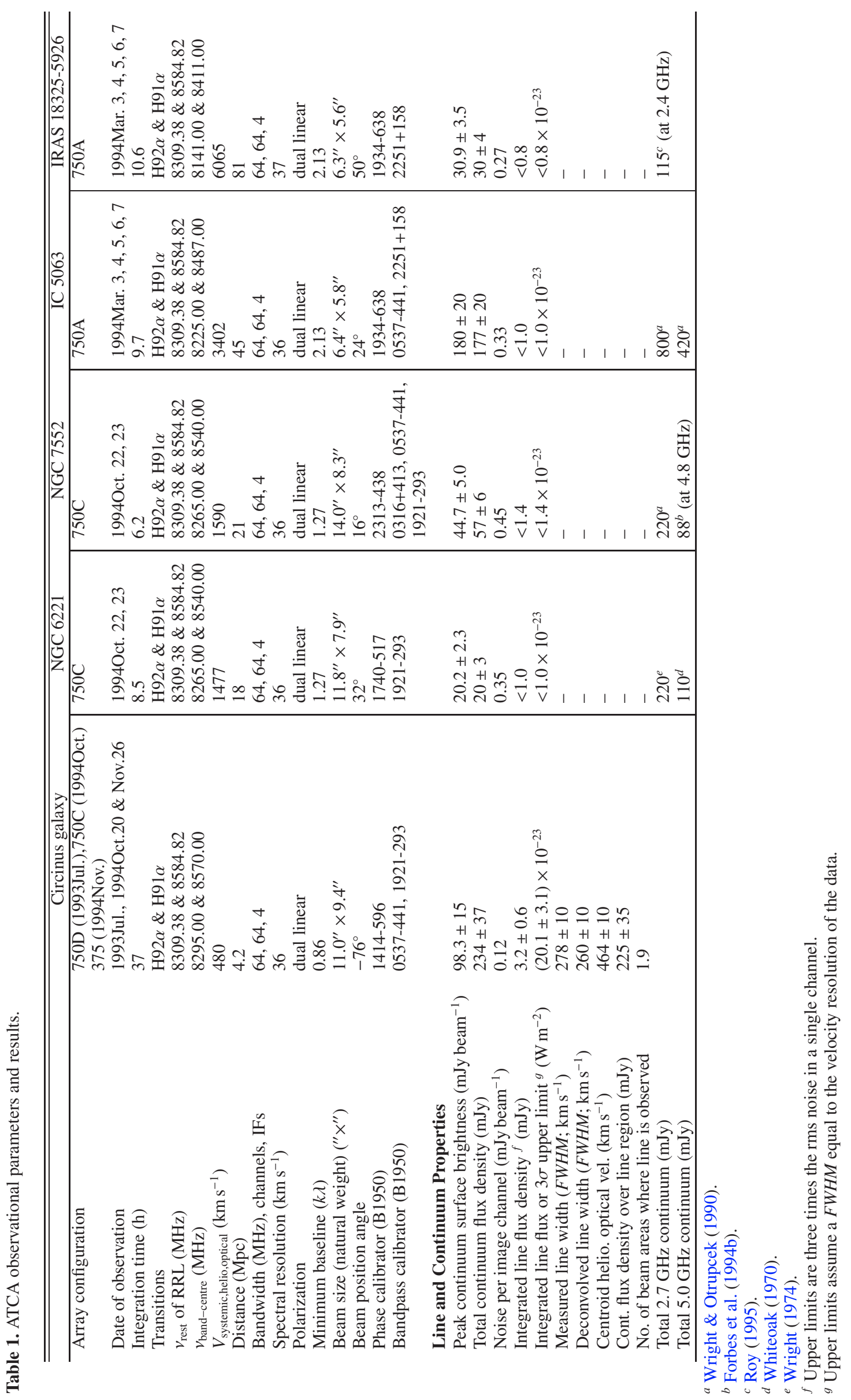




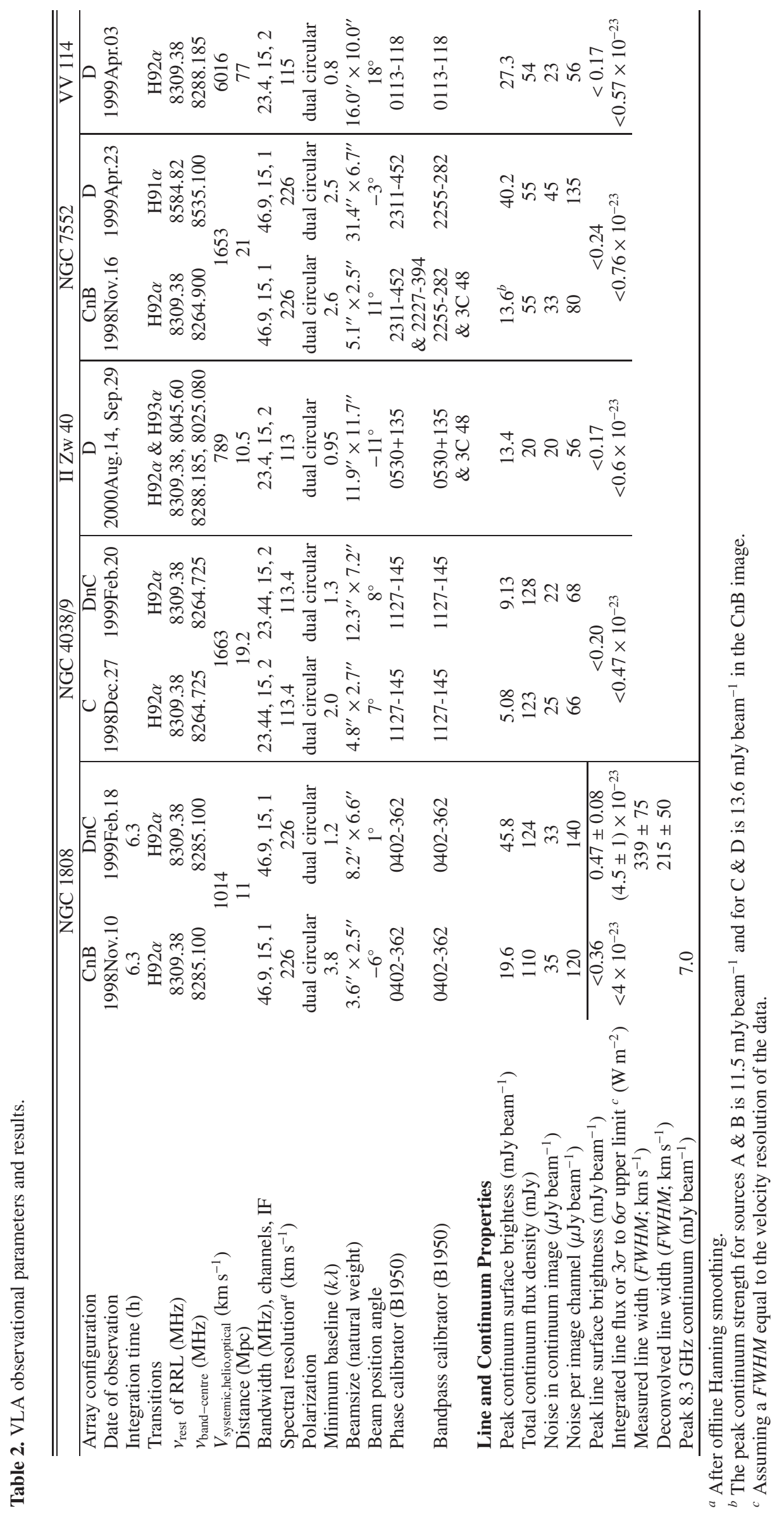




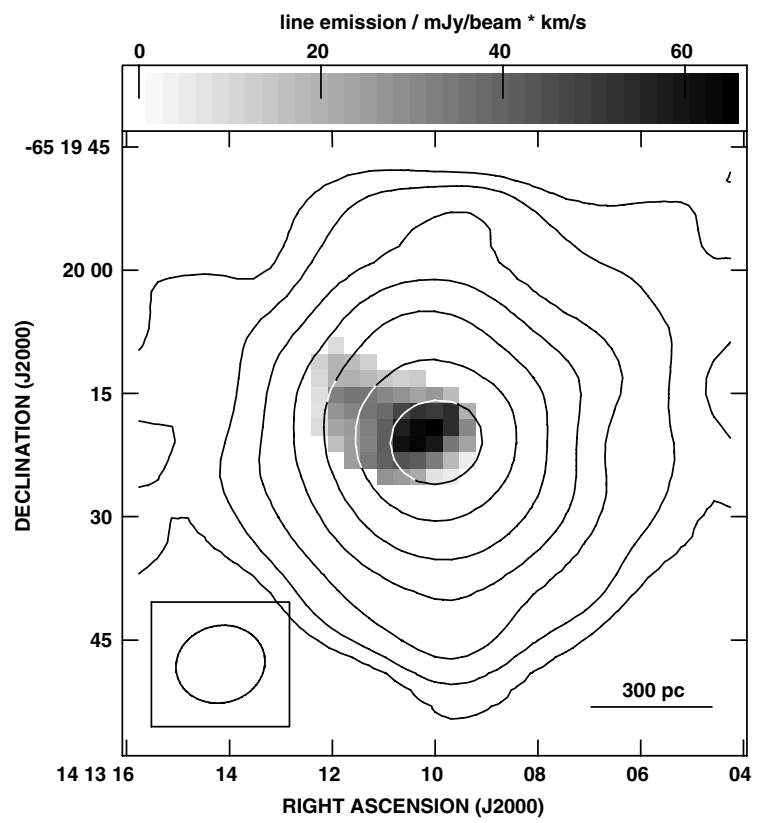

Fig. 1. ATCA $8.3 \mathrm{GHz}+8.6 \mathrm{GHz}$ continuum image of the Circinus galaxy (contours) superimposed on the grey scale zerothmoment image showing H91 $\alpha+\mathrm{H} 92 \alpha$ line emission. Peak continuum brightness is $98.5 \mathrm{mJy}_{\text {beam }}^{-1}$ and contours are at $-4,-2$, $-1,1,2,4,8,16,32$, and $64 \mathrm{mJy}_{\text {beam }^{-1}}$. Grey-scale peak = $65 \mathrm{mJy}$ beam $^{-1} \mathrm{~km} \mathrm{~s}^{-1}$. Beamsize is $11.0^{\prime \prime} \times 9.4^{\prime \prime}$ at a PA of $-76^{\circ}$, rms noise is $0.12 \mathrm{mJy}_{\text {beam }}{ }^{-1}$ channel $^{-1}$.

show the south-west side receding. The gradient direction of the RRL emission is the same as that of the molecular outflow seen by Elmouttie et al. (1997) and studied by Curran et al. (1999). Thus, the H92 $\alpha$ emitting gas is most likely associated with the outflow.

The velocity span of the signal in the position-velocity diagram $\left(130 \mathrm{~km} \mathrm{~s}^{-1}\right)$ is half as wide as the line integrated over a $38^{\prime \prime} \times 38^{\prime \prime}$ region in Fig. $2\left(260 \mathrm{~km} \mathrm{~s}^{-1}\right)$. This indicates that there is extended low-level RRL emission outside the region in the position-velocity diagram, i.e. at higher or lower declination. The line width integrated over the large area $\left(260 \mathrm{~km} \mathrm{~s}^{-1}\right)$ is comparable to that of the molecular outflow $\left( \pm 190 \mathrm{~km} \mathrm{~s}^{-1}\right.$; Curran et al. 1999) and of the ionized outflow $\left(180 \mathrm{~km} \mathrm{~s}^{-1}\right.$; Veilleux \& Bland-Hawthorn 1997) and is much larger than the width of the $\mathrm{Br} \gamma$ and $\mathrm{H}_{2}$ emission $\left(30 \mathrm{~km} \mathrm{~s}^{-1}\right)$ seen on $8 \mathrm{pc}$ scale (Müller Sanchez et al. 2006), further supporting the association of the RRL-emitting gas with the outflow.

\subsection{Modelling the ionized gas}

Two types of models have been considered for the RRL emission from the nuclei of external galaxies: one based on a uniform slab of ionized gas and the other based on a collection of compact H II regions. Such models have been discussed by Anantharamaiah et al. (2000) and references therein, and are documented in detail by Mohan (2002). These models take as constraints the integrated RRL strength at one or more frequencies, the radio continuum spectrum and the geometry of the line emitting region.

For modelling the RRL emission from the Circinus galaxy, we used the observed line strength $(3.2 \mathrm{mJy})$, line width $\left(260 \mathrm{~km} \mathrm{~s}^{-1}\right)$, size of the line-emitting region $(250 \mathrm{pc})$, continuum emission $(225 \mathrm{mJy})$, and spectral index $(-0.65)$ to constrain conditions in the ionized gas.

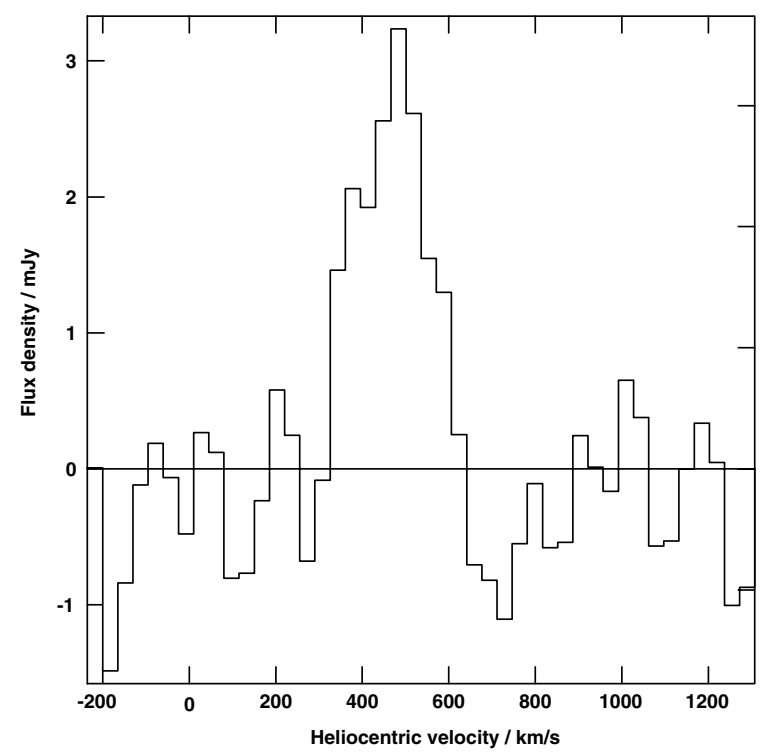

Fig. 2. ATCA H91 $\alpha+\mathrm{H} 92 \alpha$ line profile integrated over the lineemitting region in the Circinus galaxy. Region of integration is a box of size $38^{\prime \prime} \times 38^{\prime \prime}$ centred on RA $141310.01 \mathrm{dec}-652019.1$.

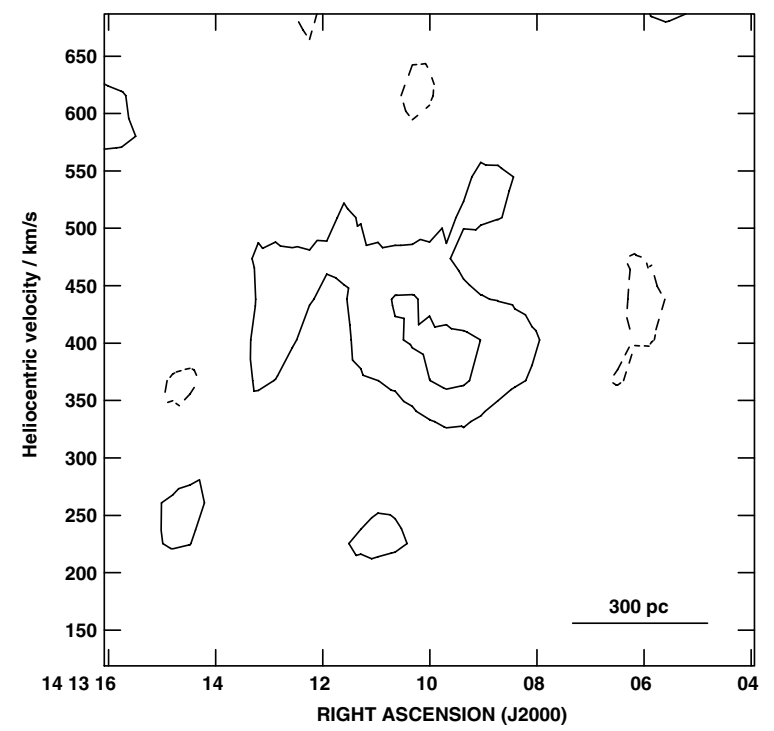

Fig. 3. ATCA position-velocity diagram of $\mathrm{H} 91 \alpha+\mathrm{H} 92 \alpha$ emission in the Circinus galaxy along a slice at constant declination $\left(-65^{\circ} 20^{\prime} 19.1^{\prime \prime}\right)$ through the maximum of the RRL emission, showing possible rotation. Beamsize is $11.0^{\prime \prime} \times 9.4^{\prime \prime}$ at a PA of $-76^{\circ}$, giving $10.6^{\prime \prime}$ resolution in RA, $36 \mathrm{~km} \mathrm{~s}^{-1}$ in velocity (no Hanning smoothing), and averaging over $9.8^{\prime \prime}$ in declination.

We used the collection of $\mathrm{H}$ II regions model from Anantharamaiah et al. (1993). We considered a grid of models with electron temperature, $T_{\mathrm{e}}$, in the range $1000 \mathrm{~K}$ to $12500 \mathrm{~K}$, electron density, $n_{\mathrm{e}}$, in the range $10 \mathrm{~cm}^{-3}$ to $10^{6} \mathrm{~cm}^{-3}$, and effective diameter of the line-emitting gas $\left(\sim\right.$ volume $\left.^{1 / 3}\right), l$, in the range $0.01 \mathrm{pc}$ to $100 \mathrm{pc}$.

We found that $3 \times 10^{3} M_{\odot}$ to $1 \times 10^{6} M_{\odot}$ of ionized gas with $T_{\mathrm{e}} \sim 5000 \mathrm{~K}, n_{\mathrm{e}} \sim 500 \mathrm{~cm}^{-3}$ to $5 \times 10^{4} \mathrm{~cm}^{-3}$, and with a total effective diameter of the line-emitting gas of $3 \mathrm{pc}$ to $50 \mathrm{pc}$ produced good matches to the line and continuum emission. We found that almost all of the RRL emission is due to stimulated emission amplifying the non-thermal continuum. The fraction of stimulated emission was around $90 \%$ in all models. Parameters derived for typical allowed models are given in Table 3 . The 
allowed range of values is large since we are using a single line strength measurement to constrain multiple parameters. Much tighter constraints would come from a multi-transition study, as was done for Arp 220 by Anantharamaiah et al. (2000).

The inferred mass of ionized gas and the Lyman continuum flux required to maintain the ionization are summarized in Table 3. The flux is equivalent to the Lyman continuum output of 300 to 9000 stars of type O5, which infers a star-formation rate of $0.2 M_{\odot} \mathrm{yr}^{-1}$ to $6 M_{\odot} \mathrm{yr}^{-1}$ when averaged over the lifetime of OB stars.

This can be compared to star formation rates derived from other indicators following Hopkins et al. (2003). Taking the peak $1.4 \mathrm{GHz}$ continuum surface brightness of $445 \mathrm{mJy}^{\text {beam }}{ }^{-1}$ in the $20^{\prime \prime} \times 19^{\prime \prime}$ beam of the ATCA at the nucleus of Circinus (Elmouttie et al. 1998b) yields a $1.4 \mathrm{GHz}$ luminosity in the central $400 \mathrm{pc}$ diameter of $9.4 \times 10^{20} \mathrm{~W} \mathrm{~m}^{-2}$ and a corresponding star formation rate of $0.86 M_{\odot} \mathrm{yr}^{-1}$. The IRAS $60 \mu \mathrm{m}$ and $100 \mu \mathrm{m}$ flux densitites yield a far-infrared (FIR) star formation rate of $0.17 M_{\odot} \mathrm{yr}^{-1}$. The $\mathrm{H} \alpha$-based star formation rate could not be estimated due to a lack of published $\mathrm{H} \alpha$ spectrophotometry. The $U$-band magnitude of 12.87 (de Vaucouleurs 1991) along with the Balmer decrement from Oliva et al. (1994) yields a U-band star formation rate of $1.63 M_{\odot} \mathrm{yr}^{-1}$. These estimates all agree well with the star formation rate of $0.2 M_{\odot} \mathrm{yr}^{-1}$ to $6 M_{\odot} \mathrm{yr}^{-1}$ estimated from the RRL emission.

\section{NGC 1808}

NGC 1808, at a distance of $11 \mathrm{Mpc}$, is a spiral galaxy (Sbc pec) undergoing a starburst, with a FIR luminosity of $2 \times 10^{10} L_{\odot}$. This galaxy exhibits a bright nucleus, a bar, several optical hotspots (Sersic \& Pastoriza 1965), and dusty radial filaments over the central few kpc (Véron-Cetty \& Véron 1985). The peculiar morphology and the starburst activity are attributed to tidal interactions with the companion NGC 1792 (Dahlem et al. 1990). H I observations show a strong outflow (Koribalski et al. 1993a), co-spatial with the dusty filaments. There is evidence both for and against Seyfert activity at the nucleus (Forbes et al. 1992; Kotilainen et al. 1996). CO and H I imaging (Dahlem et al. 1990; Koribalski et al. 1993b; Koribalski et al. 1996) shows a central concentration of gas surrounded by a $\sim 25^{\prime \prime}$ rotating molecular ring, believed to be coincident with the inner Lindblad resonance of the kpc-scale bar. There is also evidence for non-circular gas motion (Saikia et al. 1990). High resolution radio continuum images (Saikia et al. 1990; Collison et al. 1994) show that the central kpc contains a population of compact sources. NIR continuum and line imaging at $1.8^{\prime \prime}$ (Krabbe et al. 1994) and 1.0" (Kotilainen et al. 1996) shows a number of Bry, [Fe II], and $\mathrm{H}_{2}$ knots. The $\mathrm{Br} \gamma$ and radio continuum knots are spatially correlated at a resolution of $1.8^{\prime \prime}$ but are not exactly coincident at a higher resolution of $1.0^{\prime \prime}$. There is no correlation between the positions of the optical hotspots and the radio/Br $\gamma$ knots, hence the former seem to trace regions of low dust extinction rather than regions of star formation. Most of the radio knots have a steep spectral index and are probably SNRs with a thermal fraction not exceeding $\sim 30 \%$ at $\mathrm{cm}$ wavelengths. Using spectrophotometric models, Krabbe et al. (1994) and Kotilainen et al. (1996) derive an age of greater than $40 \mathrm{Myr}$ to $50 \mathrm{Myr}$ for the nuclear starburst and much younger ages of less than $10 \mathrm{Myr}$ to $15 \mathrm{Myr}$ for the circumnuclear hotspots. Tacconi-Garman et al. (1996) imaged the galaxy in the NIR $K$ band at $0.6^{\prime \prime}$ resolution and detected a number of unresolved sources, believed to be young massive super star clusters. Assuming a decaying star-formation rate, they derive ages similar to the earlier work for the nucleus

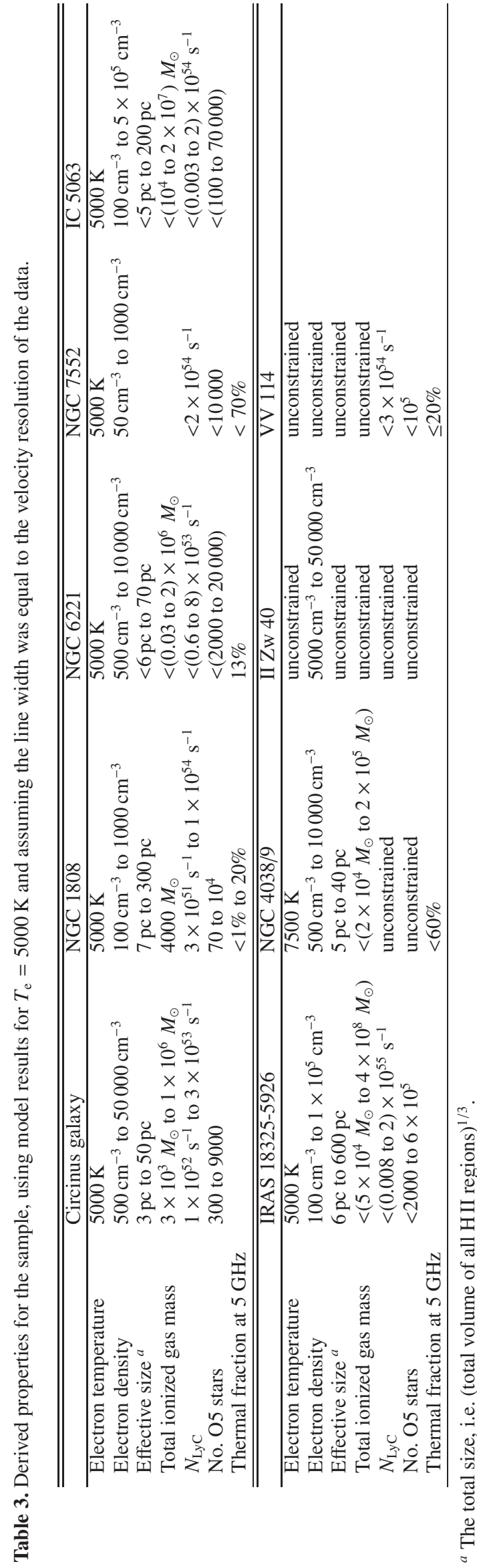




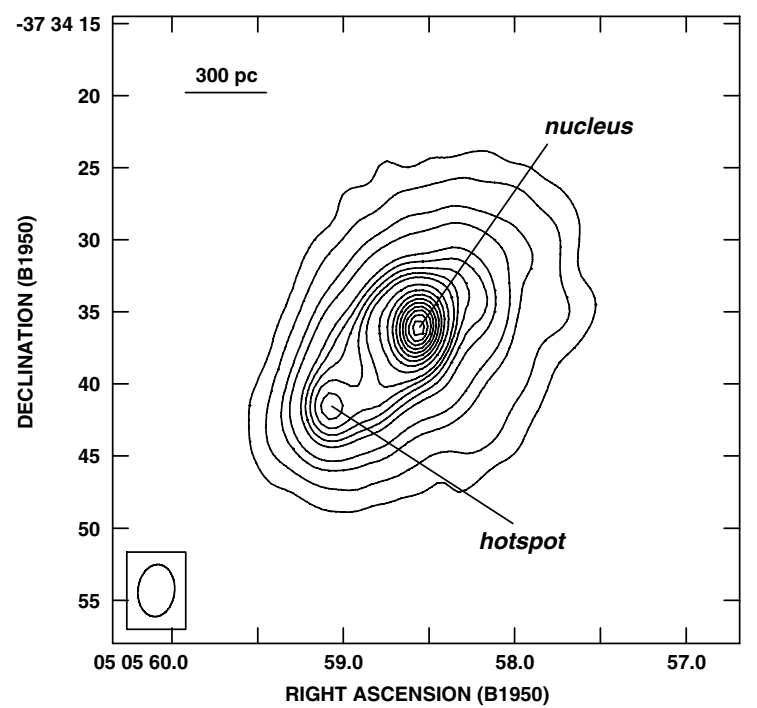

Fig. 4. 8.3 GHz continuum images of NGC 1808 with the VLA in CnB configuration. The synthesized beam is $3.6^{\prime \prime} \times 2.5^{\prime \prime}$ at a PA of $-6^{\circ}$. The rms noise is $35 \mu \mathrm{Jy}^{\mathrm{beam}}{ }^{-1}$ and the contour levels plotted are $(10,20$, $40,60,100,120,140,160,180,220,260,300,340,380,420,460,500$, $540) \times$ rms.

and the hotspots. However, they also derive a much larger age of $\sim 200$ Myr for all of the sources assuming a history of continuous star formation.

\subsection{Results}

The brightest continuum source seen in Fig. 4 is the nucleus, and the second brightest, labelled "hotspot" was seen previously in the $1.4 \mathrm{GHz}$ image of Saikia et al. (1990). Based on the $1.4 \mathrm{GHz}$ and $8.3 \mathrm{GHz}$ continuum flux densitites measured with a 3.1" beam, the spectral indices of the nucleus and the hotspot are calculated to be -0.57 and -0.65 respectively. Higher resolution (sub-arcsecond) images show that the hotspot consists of two compact distinct sources. These are referred to as A10 and A11 by Collison et al. (1994). Though diffuse $\mathrm{Br} \gamma$ emission is seen in a region encompassing both sources, only a single $\mathrm{Br} \gamma$ knot is seen in this region and it lies very close to A10 (Krabbe et al. 1994; Kotilainen et al. 1996). Hence there seems to be more thermal gas towards A10 than A11, even allowing for dust extinction. The radio continuum emission from A10 is weaker than from A11 in the high resolution images. However, images at a lower resolution of $3.1^{\prime \prime}$ at $8.3 \mathrm{GHz}$ (this work) and $1.4 \mathrm{GHz}$ (Saikia et al. 1990) show that the smooth emission peaks much nearer the position of A10, indicating the presence of substantial diffuse emission around this source.

H92 $\alpha$ line emission integrated over the central $18^{\prime \prime} \times 20^{\prime \prime}$ was detected in the DnC configuration data (Fig. 5), extending between $750 \mathrm{~km} \mathrm{~s}^{-1}$ and $1100 \mathrm{~km} \mathrm{~s}^{-1}$. This velocity range is consistent with the HI velocity range of (800 to 1200$) \mathrm{km} \mathrm{s}^{-1}$ (Koribalski et al. 1993a), considering the low signal-to-noise ratio of the RRL detection. The observed FWHM of the line is $(339 \pm 75) \mathrm{km} \mathrm{s}^{-1}$ and the deconvolved line width, after correcting for offline Hanning smoothing and spectral resolution, is about $210 \mathrm{~km} \mathrm{~s}^{-1}$. The peak line emission $\left(470 \mu \mathrm{Jy}_{\text {beam }}{ }^{-1}\right)$ is only $3.9 \sigma$.

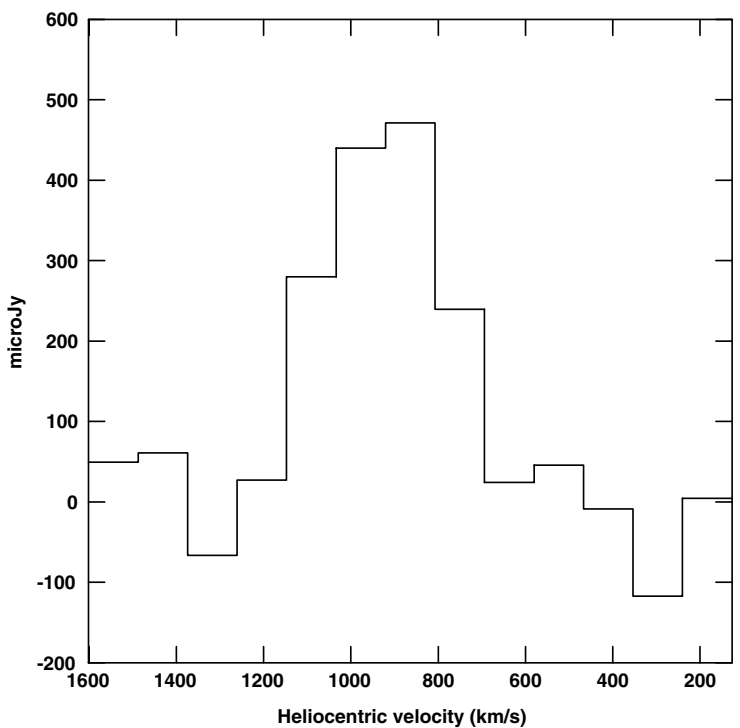

Fig. 5. The Hanning smoothed H92 $\alpha$ spectrum in NGC 1808 for the DnC configuration data, integrated over a box of size of $18^{\prime \prime} \times 20^{\prime \prime}$ centred on RA $050558.58 \mathrm{dec}-373436.5$. The synthesized beam is $8.2^{\prime \prime} \times 6.6^{\prime \prime}$ and the $1 \sigma$ noise in the channel image is $140 \mu \mathrm{Jy}_{\text {beam }}{ }^{-1}$. The peak of the line integrated over the region is $0.47 \mathrm{mJy}$ and the continuum emission integrated over the same region is $101 \mathrm{mJy}$ giving a line-to-continuum ratio of 0.005 .

\subsection{Modelling the ionized gas}

We modelled the thermal gas in NGC 1808 using the measured continuum and line strengths at $8.3 \mathrm{GHz}$ and a spectral index of -0.7 at $8.3 \mathrm{GHz}$. We modelled the line-emitting gas as a collection of H II regions. For each combination of input parameters in a given model and assuming conservatively that the thermal gas was spread homogeneously over the entire region, we corrected the intensity of the non-thermal radiation for absorption by the thermal gas and calculated the expected free-free continuum emission from the model H II regions. The resulting free-free emission was constrained to be less than the observed thermal emission of $30 \mathrm{mJy}$, which was estimated from the total observed continuum emission of $101 \mathrm{mJy}$ assuming a thermal fraction of $30 \%$.

The allowed range of electron density is $100 \mathrm{~cm}^{-3}$ to $1000 \mathrm{~cm}^{-3}$ and the effective diameter of the line-emitting gas $\left(\sim\right.$ volume $\left.^{1 / 3}\right), l$, is in the range $7 \mathrm{pc}$ to $300 \mathrm{pc}$. The ionization rate is $3 \times 10^{51} \mathrm{~s}^{-1}$ to $1 \times 10^{54} \mathrm{~s}^{-1}$. The free-free flux density at $8.3 \mathrm{GHz}$ is $<1.0 \mathrm{mJy}$ to $20 \mathrm{mJy}$ which is $<1 \%$ to $20 \%$ of the total continuum.

Model solutions were calculated for $T_{\mathrm{e}}$ of $5000 \mathrm{~K}, 7500 \mathrm{~K}$, and $10000 \mathrm{~K}$. The derived parameters varied by much less than the range in the allowed parameter values for any given $T_{\mathrm{e}}$ value. Thus, we present parameters for $T_{\mathrm{e}}=5000 \mathrm{~K}$, but the values for $T_{\mathrm{e}}=7500 \mathrm{~K}$ or $10000 \mathrm{~K}$ would be almost the same. The fraction of stimulated emission, as for the Circinus galaxy, was around $90 \%$ in all models.

Using the derived value of $N_{\mathrm{Lyc}}=3 \times 10^{51} \mathrm{~s}^{-1}$ to $1 \times 10^{54} \mathrm{~s}^{-1}$, the $\mathrm{Br} \gamma$ flux is expected to be $3 \times 10^{-18} \mathrm{~W} \mathrm{~m}^{-2}$ to $9 \times 10^{-16} \mathrm{~W} \mathrm{~m}^{-2}$. For comparison, Krabbe et al. (1994) measured the $\operatorname{Br} \gamma$ flux from the hotspot region to be $2.6 \times 10^{-17} \mathrm{~W} \mathrm{~m}^{-2}$ and the data of Kotilainen et al. (1996) yield $1.6 \times 10^{-17} \mathrm{~W} \mathrm{~m}^{-2}$. Both measurements are within the broad range expected given the (extinctionfree) RRL strength.

To estimate the effect of small changes in the $b_{\mathrm{n}}$ and $\beta$ coefficients on the derived physical parameters of the ionized gas, 

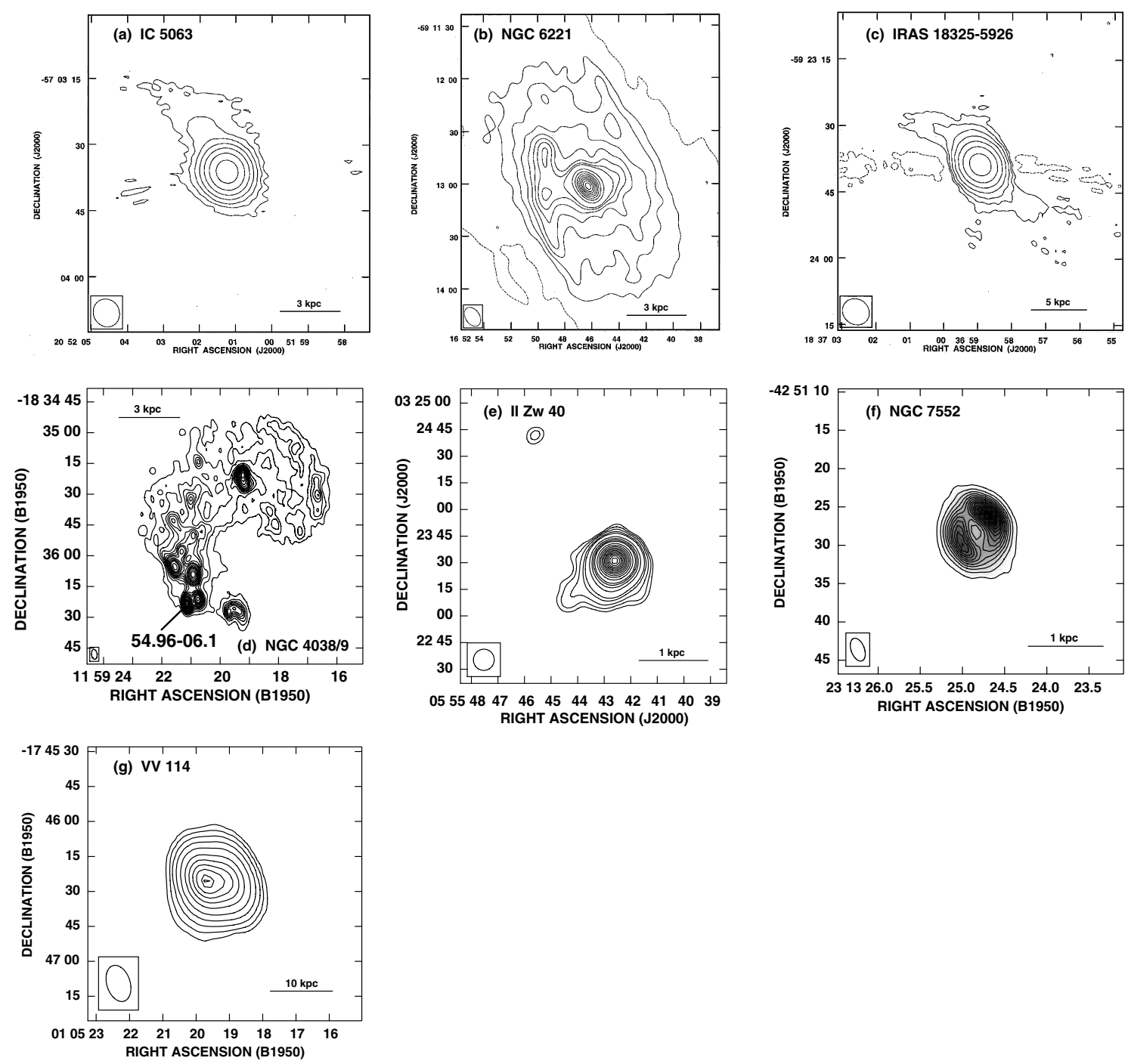

Fig. 6. a) $8.4 \mathrm{GHz}$ continuum image of IC 5063 using the ATCA. The synthesized beam is $6.4^{\prime \prime} \times 5.8^{\prime \prime}$ at a PA of $24^{\circ}$. The peak brightness is $180 \mathrm{mJy}$ beam $^{-1}$ and the contour levels are at $-1.8,1.8,3.6,7.2,14.4,28.8$ and $57.5 \mathrm{mJy}_{\text {beam }}^{-1}$. b) $8.4 \mathrm{GHz}$ continuum image of NGC 6221 using the ATCA. The synthesized beam is $11.8^{\prime \prime} \times 7.9^{\prime \prime}$ at a PA of $32^{\circ}$. The peak brightness is $20.2 \mathrm{mJy} \mathrm{beam}^{-1}$ and the contour levels are at -0.4 , $0.4,0.8,1.2,1.6,2.0,2.4,2.8,4.8,6.9,8.9,10.9,14.9,14.9,17.0$ and 19.0 mJy beam $^{-1}$. c) $8.3 \mathrm{GHz}$ continuum image of IRAS $18325-5926$ using the ATCA. The synthesized beam is $6.3^{\prime \prime} \times 5.6^{\prime \prime}$ at a PA of $50^{\circ}$. The peak brightness is $30.9 \mathrm{mJy}$ beam $^{-1}$ and the contour levels are at $-0.3,0.3$, $0.6,1.2,2.5,4.9,9.9$ and $19.8 \mathrm{mJy}_{\text {beam }}^{-1}$. d) $8.3 \mathrm{GHz}$ VLA continuum image of NGC 4038/9 using the C configuration. The rms noise in the image is $25 \mu \mathrm{Jy}_{\text {beam }}{ }^{-1}$ and the contour levels are $(5,10,15,20,25,30,35,40,50,60,70,80,100,120,140,160,180,200) \times$ rms in image. The synthesized beam is $4.8^{\prime \prime} \times 2.7^{\prime \prime}$ at a PA of $7^{\circ}$. e) $8.3 \mathrm{GHz}$ continuum image of II Zw 40 using the VLA in the D configuration. The synthesized beam is $11.9^{\prime \prime} \times 11.7^{\prime \prime}$ at a PA of $-11^{\circ}$ and the rms in the image is $20 \mu \mathrm{Jy} \mathrm{beam}^{-1}$. The contour levels are $(5,7,10,15,20,40,60,80,100,150,200$, $250,300,350,400,450,500,550,600,650)$ times the rms. f) $8.3 \mathrm{GHz}$ continuum image of NGC 7552 using the VLA in the CnB configuration with uniform weighting. The rms in the image is $58 \mu \mathrm{Jy}_{\text {beam }}{ }^{-1}$ and the contour levels are $(10,20,30,40,50,60,70,80,90,100,110,120$, 130) $\times$ rms. The synthesized beam is $3.1^{\prime \prime} \times 1.7^{\prime \prime}$ at a PA of $20^{\circ}$. g) $8.3 \mathrm{GHz}$ continuum image of VV 114 in the D configuration. The contour levels are $(10,20,40,80,160,320,500,700,900,1100,1170) \times \mathrm{rms}$ in the image, which is $23 \mu \mathrm{Jy} \mathrm{beam}^{-1}$. The synthesized beam is $16.0^{\prime \prime} \times 10.0^{\prime \prime}$ at a PA of $18^{\circ}$.

which is a concern since the line emission is strongly affected by stimulated emission, we note that when we ran models for adjacent lines having roughly the same observed parameters, we obtained essentially the same values of derived parameters and conclude that the effects of changes are small.

Parameters derived for typical allowed models are given in Table 3 .

\section{The non-detections}

The H92 $\alpha$ line was not detected in the other galaxies observed with the ATCA (NGC 6221, IC 5063, IRAS 18325-5926, and NGC 7552) or with the VLA (NGC 4038/9, II Zw 40, NGC 7552, and VV 114). Upper limits were $0.8 \mathrm{mJy}^{\text {beam }}{ }^{-1}$ to $1.4 \mathrm{mJy}_{\text {beam }}^{-1}(3 \sigma)$ for the ATCA and $0.17 \mathrm{mJy} \mathrm{beam}^{-1}$ to 0.24 mJy beam $^{-1}(3 \sigma)$ for the VLA observations (Tables 1 and 2). The continuum images are shown in Fig. 6.

IC 5063 is classed as a NLRG, having $5 \times 10^{23} \mathrm{~W} \mathrm{~Hz}^{-1}$ at $1.4 \mathrm{GHz}$. The nuclear component was resolved into a $4^{\prime \prime}$-long linear triple structure at $8.6 \mathrm{GHz}$ by Morganti et al. (1998). Our $8.4 \mathrm{GHz}$ image (Fig. 6a) with the larger beam of $6.4^{\prime \prime} \times 5.8^{\prime \prime} \mathrm{did}$ not resolve the jet, as expected.

We used the upper limits on the H91 $\alpha+\mathrm{H} 92 \alpha$ line emission to constrain the properties of the nuclear emission-line regions. 
We assumed that the line $F W H M$ was the same as the velocity resolution of the data (from Tables 1 and 2). We modelled each source as a collection of uniform-density spherical H II regions. The model solutions were constrained to produce an $8.3 \mathrm{GHz}$ free-free emission equal to $30 \%$ of the continuum flux density integrated over the central region and an $\mathrm{H} 92 \alpha$ line emission equal to the observed upper limit. The derived value for the gas density varied between $100 \mathrm{~cm}^{-3}$ and $5 \times 10^{5} \mathrm{~cm}^{-3}$ and the total effective diameter of the H II regions varied between $5 \mathrm{pc}$ to $200 \mathrm{pc}$ with the effective size decreasing with increasing density. The total flux of ionizing photons required to maintain such nebulae on the limit of our detectability ranged between $3 \times 10^{51} \mathrm{~s}^{-1}$ and $2 \times 10^{54} \mathrm{~s}^{-1}$ depending on density and size of the individual HII regions. This provides upper limits to the number of O5 stars powering the ionization in the range 100 to 70000 , with the higher values corresponding to those models whose combination of parameters yield high free-free continuum emission from the ionized gas.

Parameters derived for typical allowed models are given in Table 3.

NGC 6221 contains two emission-line regions in the nucleus - a low-excitation H II region probably photoionized by hot, young stars and a faint high-excitation region with relatively broad emission lines characteristic of Seyfert 2 nuclei (Véron et al. 1981). Our continuum image at $8.3 \mathrm{GHz}$ (Fig. 6b) shows emission from the nucleus, bar and spiral arms, consistent with that seen at $1.4 \mathrm{GHz}$ by Koribalski \& Dickey (2004).

We used the upper limits on the H91 $\alpha+\mathrm{H} 92 \alpha$ line emission to constrain the properties of the nuclear emission-line regions following the approach described for IC 5063. Parameters derived for typical allowed models are given in Table 3.

IRAS 18325-5926 is an ultraluminous infrared galaxy with a compact central radio component ( size $\leq 0.1^{\prime \prime}$ ) which accounts for one fifth of the total emission at $2.3 \mathrm{GHz}$ (Roy et al. 1994). Our $8.3 \mathrm{GHz}$ image in Fig. $6 \mathrm{c}$ is consistent with that of Bransford et al. (1998), showing an unresolved component with no significant extended structure. The flux density in our observation, $30 \mathrm{mJy} \pm 4 \mathrm{mJy}$, is lower than the $36.8 \mathrm{mJy} \pm 0.3 \mathrm{mJy}$ measured by Bransford et al. (1998) (after interpolating between their 4.8 $\mathrm{GHz}$ and $8.6 \mathrm{GHz}$ measurements). The source might therefore have varied between 1993 Jul. and 1995 Nov.

We used the upper limits on the H91 $\alpha+\mathrm{H} 92 \alpha$ line emission to constrain the properties of the nuclear emission-line regions following the approach described for IC 5063. Parameters derived for typical allowed models are given in Table 3 .

NGC 4038/9 is a pair of interacting galaxies with extended tidal tails and numerous star clusters, including super star clusters (Whitmore \& Schweizer 1995; Whitmore et al. 1999). Neff \& Ulvestad (2000) imaged the multi-frequency radio continuum emission in this galaxy at high resolution (1.0" to 2.6") and identified a host of compact sources, some of which exhibit a spectral index flatter than -0.4 , indicating a high thermal gas fraction. Our continuum image (Fig. 6d) is consistent with theirs, showing the same collection of compact sources immersed in diffuse emission. Our image is at lower resolution and has a factor two higher noise.

We used the upper limit on the RRL emission to constrain the properties of the strongest thermal source 54.96-06.1 (see Fig. 6d). Neff \& Ulvestad (2000) determined the diameter of this source to be $70 \mathrm{pc}\left(0.7^{\prime \prime}\right)$ and derived an rms density of $\sim 400 \mathrm{~cm}^{-3}$.

We modelled this source as a collection of uniform-density spherical H II regions, constrained by the H92 $\alpha$ upper limit, the $8.3 \mathrm{GHz}$ flux density of Neff \& Ulvestad (2000) as an upper limit, the observed $4.8 \mathrm{GHz}$ to $8.3 \mathrm{GHz}$ spectral index, and the infra-red excess (IRE), $L_{\mathrm{FIR}} / N_{\mathrm{Lyc}}$ (derived from $L_{\mathrm{FIR}}=$ $1.3 \times 10^{36} \mathrm{~W}$; Neff \& Ulvestad (2000) and the $N_{\text {Lyc }}$ of the models) to exceed unity. No solutions were obtained for the thermal fraction of the $8.3 \mathrm{GHz}$ continuum $f_{\text {th }}>0.6$ if the RRL constraint was included. The derived values for the gas density and the size of the $\mathrm{H}$ II region varied between $5 \times 10^{2} \mathrm{~cm}^{-3}$ to $10^{4} \mathrm{~cm}^{-3}$ and $40 \mathrm{pc}$ to $5 \mathrm{pc}$, and the IRE was 6 to 10 . The H92 $\alpha$ line strength is $\sim 80 \%$ of the $3 \sigma$ upper limit if the thermal fraction is not less than $30 \%$ and hence might be detectable with sensitive observations. However, the observed flat spectral index of $-0.26 \pm 0.13$ cannot be reconciled with the low derived thermal fraction unless there is additional low density thermal gas which does not emit 8.3 GHz RRL. It is surprising that we do not detect RRL emission from other regions in the galaxy. Since it is unlikely that the starburst has declined rapidly over the last million years, a search for RRLs at much lower resolution, possibly using the GBT, might prove more fruitful.

II Zw 40 was imaged by Beck et al. (2002) at $15 \mathrm{GHz}$ using the VLA and discovered four unresolved objects. They identified the continuum emission as free-free and suggested that these are similar to the young "supernebulae" discovered in NGC 5253 (Turner et al. 2000) and He 2-10 (Kobulnicky \& Johnson 1999). They show that these supernebulae account for almost all of the $12 \mu \mathrm{m}$ emission from the inner core. We show our continuum image in Fig. 6e, which, as expected due to the lower resolution, shows the multiple sources as a single unresolved component.

We used the upper limits on the H92 $\alpha+\mathrm{H} 93 \alpha$ line emission with a model consisting of an individual spherical H II region of uniform density, constrained to produce a $15 \mathrm{GHz}$ continuum free-free emission of $0.6 \mathrm{mJy}$. Solutions were obtained for $n_{\mathrm{e}} \geq 5000 \mathrm{~cm}^{-3}$. The expected $8.3 \mathrm{GHz} \mathrm{H} 92 \alpha$ line flux for the various model solutions for all four objects is $60 \%$ to $70 \%$ of the observed $3 \sigma$ upper limit for densities between $5000 \mathrm{~cm}^{-3}$ and $10000 \mathrm{~cm}^{-3}$, is about $40 \%$ for $n_{\mathrm{e}} \sim 50000 \mathrm{~cm}^{-3}$ and decreases further for higher densities.

NGC 7552 contains three star forming rings of radii $1.0 \mathrm{kpc}$, $1.9 \mathrm{kpc}$, and $3.4 \mathrm{kpc}$ at the co-rotation and Lindblad resonance radii of the bar. The inner-most ring is seen in radio continuum (Forbes et al. 1994a). Our continuum image (Fig. 6f) shows the ring structure seen previously by Forbes et al. A number of $\mathrm{Br} \gamma$ knots lie along the inner ring which show weak correlation with radio knots, but these are not spatially resolved in our observations.

We used the upper limits on the H91 $\alpha+\mathrm{H} 92 \alpha$ line emission to constrain the properties of the nuclear emission-line regions. Parameters derived for typical allowed models are given in Table 3.

VV 114 is undergoing a merger and the two nuclei VV 114E and VV $114 \mathrm{~W}$ are separated by $15^{\prime \prime}(5.6 \mathrm{kpc})$. The E and $\mathrm{W}$ components are barely resolved in the continuum image shown in Fig. 6g.

The model for the thermal component of VV $114 \mathrm{E}$ was constrained by the upper limit to the H92 $\alpha$ line emission and a free-free emission of $f_{\text {th }} \times S_{\mathrm{c}}$ where $S_{\mathrm{c}}$ is $27.2 \mathrm{mJy}$ at $8.3 \mathrm{GHz}$. The IRE was constrained to be greater than unity by assuming that the FIR luminosity of VV $114 \mathrm{E}$ is $1 \times 10^{11} L_{\odot}$. Consistent solutions were obtained only for a thermal fraction $\leq 20 \%$, i.e., $S_{\mathrm{c}} \leq 5.4 \mathrm{mJy}$. If this thermal gas is optically thin at $8.3 \mathrm{GHz}$, then the ionization rate is $<3 \times 10^{54} \mathrm{~s}^{-1}$. 


\section{Conclusions}

We have detected $\mathrm{H} 91 \alpha$ or $\mathrm{H} 92 \alpha$ lines in emission in the Circinus galaxy and NGC 1808 with integrated flux densities of $3.2 \mathrm{mJy}$ and $0.47 \mathrm{mJy}$ respectively using the ATCA and the VLA. We established upper limits on the RRL strength in NGC 4038/9, II Zw 40, NGC 6221, NGC 7552, IRAS 18325 5926, IC 5063, and VV 114. The detected line strengths infer ionized gas masses of $3000 M_{\odot}$ to $10^{6} M_{\odot}$ in the Circinus galaxy and $10^{4} M_{\odot}$ in NGC 1808 , and corresponding star formation rates of $0.2 M_{\odot} \mathrm{yr}^{-1}$ to $6 M_{\odot} \mathrm{yr}^{-1}$ and $0.3 M_{\odot} \mathrm{yr}^{-1}$ to $0.6 M_{\odot} \mathrm{yr}^{-1}$, depending on the model conditions. The star formation rate estimated from the RRL detection in Circinus agrees well with rates estimated from radio, FIR, and $U$-band luminosities using previously-calibrated relations.

Since the detectability of RRL emission depends so sensitively on the gas density, future prospects for using RRL emission as diagnostics of star formation activity are good if one observes multiple transitions and includes higher frequencies. Multi-transition studies are important to provide sensitivity to a wide range of gas densities and the details of which particular transitions are detected provide the density distribution of the ionized gas. The line strengths have been predicted and found to increase with frequency, making studies at $22 \mathrm{GHz}, 43 \mathrm{GHz}$, and $86 \mathrm{GHz}$ attractive due to their larger line-to-continuum ratios. Further, the interpretation of line strengths at higher frequency is simplified since stimulated emission is much less important towards high frequency, giving a direct relationship between RRL emission strength and the ionized gas mass, and hence to the star formation rate.

Acknowledgements. The National Radio Astronomy Observatory is a facility of the National Science Foundation operated under cooperative agreement by Associated Universities, Inc. The Australia Telescope Compact Array is part of the Australia Telescope, which is funded by the Commonwealth of Australia for operation as a National Facility managed by CSIRO.

\section{References}

Anantharamaiah, K. R., \& Goss, W. M. 1997, RMxAC, 6, 58

Anantharamaiah, K. R., Zhao, J. H., Goss, W. M., \& Viallefond, F. 1993, ApJ, 419,585

Anantharamaiah, K. R., Viallefond, F., Mohan, N. R., Goss, W. M., \& Zhao, J. H. 2000, ApJ, 537, 613

Baars J. W. M., Genzel, R., Pauliny-Toth, I. I. K., \& Witzel, A. 1977, A\&A, 61, 99

Beck, S. C., Turner, J. L., Langland-Shula, L. E., et al. 2002, AJ, 124, 2516

Bell, M. B., \& Seaquist, E. R. 1978, ApJ, 223, 378

Bell, M. B., Seaquist, E. R., Mebold, U., Reif, K., \& Shaver, P. A. 1984, A\&A, 130,1

Bransford, M. A., Appleton, P. N., Heisler, C. A., Norris, R. P., \& Marston, A. P. 1998, ApJ, 497, 133

Churchwell, E., \& Shaver, P. A. 1979, A\&A, 77, 316

Collison, P. M., Saikia, D. J., Pedlar, A., Axon, D. J., \& Unger, S. W. 1994, MNRAS, 268, 203

Cornwell, T. J., Uson, J. M., \& Haddad, N. 1992, A\&A, 258, 583

Curran, S. J., Johansson, L. E., Rydbeck, G., \& Booth, R. S. 1998, A\&A, 338, 863

Curran, S. J., Rydbeck, G., Johansson, L. E. B., \& Booth, R. S. 1999, A\&A, 344, 767

Curran, S. J., Johansson, L. E. B., Bergman, P., Heikkilä, A., \& Alto, S. 2001, A\&A, 367, 457

Dahlem, M., Aalto, S., Klein, U., et al. 1990, A\&A, 240, 237

Davies, R. E., Forbes, D. A., Ryder, S., et al. 1998, MNRAS, 293, 189 de Vaucouleurs, et al. 1991, Third Reference Catalogue of Bright Galaxies, version 3.9

Elmouttie, M., Haynes, R. F., Jones, K. L., et al. 1995, MNRAS, 275, L53

Elmouttie, M., Haynes, R. F., \& Jones, K. L. 1997, PASA, 14, 140

Elmouttie, M., Koribalski, B., Gordon, S., et al. 1998a, MNRAS, 297, 49

Elmouttie, M., Haynes, R. F., Jones, K. L., Sadler, E. M., \& Ehle, M. 1998b, MNRAS, 297, 1202

Elmouttie, M., Krause, M., Haynes, R. F., \& Jones, K. L. 1998c, MNRAS, 300, 1119

Forbes, D. A., Boisson, C., \& Ward, M. J. 1992, MNRAS, 259, 293

Forbes, D. A., Kotilainen, J. K., \& Moorwood, A. F. M. 1994a, ApJ, 433, L13

Forbes, D. A., Norris, R. P., Williger, G. M., \& Smith, R. C. 1994b, AJ, 107, 984

Frater, R. H., Brooks, J. W., \& Whiteoak, J. B. 1992, JEEEA, 12, 103

Greenhill, L. J., Kondratko, P. T., Lovell, J. E. J., et al. 2003a, ApJ, 582, L11

Greenhill, L. J., Booth, R. S., Ellingsen, S. P., et al. 2003b, ApJ, 590, 162

Harnett, J. I., Whiteoak, J. B., Gardner, F. F., Reynolds, J. E., \& Tziousmis, A. 1990, MNRAS, 244, 130

Hibbard, J. E., van der Hulst, J. M., Barnes, J. E., \& Rich, R. M. 2001, AJ, 122, 2969

Hopkins, A. M., Miller, C. J., Nichol, R. C., et al. 2003, ApJ, 599, 971

Jones, K. L., Koribalski, B. S., Elmouttie, M., \& Haynes, R. F. 1999, MNRAS, 302, 649

Juraszek, S. J., Staveley-Smith, L., Kraan-Korteweg, R. C., et al. 2000, AJ, 119, 1627

Kobulnicky, H. A., \& Johnson, K. E. 1999, ApJ, 527, 154 (KJ99)

Koribalski, B., Dahlem, M., Mebold, U., \& Brinks, E. 1993a, A\&A, 268, 14

Koribalski, B., Dickey, J. M., \& Mebold, U. 1993b, ApJ, 402, L41

Koribalski, B., Dettmar, R.-J., Mebold, U., \& Wielebinski, R. 1996, A\&A, 315, 71

Koribalski, B., \& Dickey, J. M. 2004, MNRAS, 348, 1255

Kotilainen, J. K., Forbes, D. A., Moorwood, A. F. M., van der Werf, P. P., \& Ward, M. J. 1996, A\&A, 313, 771

Krabbe, A., Sternberg, A., \& Genzel, R. 1994, ApJ, 425, 72

Mohan, N. R. 2002, Ph.D. Thesis, IISc, Bangalore, India

Mohan, N. R., Anantharamaiah, K. R., \& Goss, W. M. 2001, ApJ, 557, 659

Morganti, R., Oosterloo, T., \& Tsvetanov, Z. 1998, AJ, 115, 915

Müller Sanchez, F., Davies, R. I., Eisenhauer, F., et al. 2006, A\&A, 454, 481

Neff, S. G., \& Ulvestad, J. S. 2000, AJ, 120, 670

Oliva, E., Salvati, M., Moorwood, A. F. M., \& Marconi, A. 1994, A\&A, 288, 457

Phookun, B., Anantharamaiah, K. R., \& Goss, W. M. 1998, MNRAS, 295, 156

Rodríguez-Rico, C. A., Goss, W. M., Zhao, J.-H., Gómez, Y., \& Anantharamaiah, K. R. 2006, ApJ, 644, 914

Roy, A. L. 1995, Ph.D. Thesis, University of Sydney

Roy, A. L., Norris, R. P., Kesteven, M. J., Troup, E. R., \& Reynolds, J. E. 1994, ApJ, 432, 496

Roy, A. L., Goss, W. M., Mohan, N. R., \& Anantharamaiah, K. R. 2005a, A\&A, 435,831

Roy, A. L., Goss, W. M., Mohan, N. R., Oosterloo, T., \& Anantharamaiah, K. R. 2005b, in The Evolution of Starbursts, 331st Wilhelm and Else Heraeus Seminar, 16-20 August, 2004, Bad Honnef, Germany, Susanne Huettemeister, ed. E. Manthey, D. Bomans, \& K. Weis (New York: AIP), AIP Conf. Proc., 783, 303

Saikia, D. J., Unger, S. W., Pedlar, A., et al. 1990, MNRAS, 245, 397

Seaquist, E. R., \& Bell, M. B. 1977, A\&A, 60, L1

Sersic, J. L., \& Pastoriza, M. 1965, PASP, 77, 287

Shaver, P. A. 1978, A\&A, 68, 97

Shaver, P. A., Churchwell, E., \& Rots, A. H. 1977, A\&A, 55, 435

Tacconi-Garman, L. E., Sternberg, A., \& Eckart, A. 1996, AJ, 112, 918

Turner, J. L., Beck, S. C., \& Ho, P. T. P. 2000, ApJ, 532, L109

Véron-Cetty, M. P., \& Véron, P. 1985, A\&A, 145, 425

Véron, M. P., Véron, P., \& Zuiderwijk, E. J. 1981, A\&A, 98, 34

Veilleux, S., \& Bland-Hawthorn, J. 1997, ApJ, 479, L105

Whiteoak, J. B. 1970, ApL, 5, 29

Whitmore, B. C., \& Schweizer, F. 1995, AJ, 109, 960

Whitmore, B. C., Zhang, Q., Leitherer, C., et al. 1999, AJ, 118, 1551

Wilson, A. S., Shopbell, P. L., Simpson, C., et al. 2000, AJ, 120, 1325

Wright, A. E. 1974, MNRAS, 167, 273

Wright, A. E., \& Otrupcek R. 1990, Parkes Catalog, ATNF

Zhao, J. -H., Anantharamaiah, K. R., Goss, W. M., \& Viallefond, F. 1996, ApJ, 472,54 